\title{
Preferência alimentar sanguínea de Lutzomyia whitmani (Diptera, Psychodidae) em área de transmissão de leishmaniose cutânea americana, no Estado do Maranhão, Brasil
}

\author{
Blood feeding preference of Lutzomyia whitmani (Diptera, Psychodidae) \\ in a transmission area for American cutaneous leishmaniasis \\ in the State of Maranhão, Brazil
}

\author{
Raquel Silva Fonteles ${ }^{1}$, Gabriel Costa e Vasconcelos ${ }^{1}$, Patrícia Castelo Branco Azevêdo ${ }^{1}$, \\ Gildevan Nolasco Lopes ${ }^{1}$, Jorge Luiz Pinto Moraes ${ }^{2}$, Elias Seixas Lorosa ${ }^{3}$, \\ Oliver Kuppinger ${ }^{4}$ José Manuel Macário Rebêlo ${ }^{4}$
}

\begin{abstract}
RESUMO
O objetivo deste estudo foi determinar as fontes de alimento sanguíneo de fêmeas de Lutzomyia whitmani, espécie de flebotomíneo incriminada no Maranhão como principal vetor da leishmaniose cutânea americana. Para isso, 70 fêmeas desta espécie coletadas no município de Axixá, área com um dos maiores números de casos de leishmaniose cutânea americana em humanos no Maranhão, foram analisadas utilizando a técnica da precipitina. Dos indivíduos analisados, $90 \%$ apresentaram reação a algum tipo de antissoro e dentre estes, $73 \%$ apresentaram reações do tipo simples com predominância para sangue de galinha $(22,2 \%)$, roedor $(14,3)$ e humano $(12,7 \%)$. Nas reações duplas predominaram as combinações galinha/humano (6,3\%), galinha/gambá (4,8\%), boi/humano e gambá/humano (3,2\%). Assim, concluímos que seres humanos, animais domésticos e sinantrópicos constituem fonte alimentar sanguínea para Lutzomyia whitmani podendo desempenhar um papel importante no ciclo de transmissão da leishmaniose cutânea americana explicando os casos da doença em Axixá.
\end{abstract}

Palavras-chaves: Lutzomyia whitmani. Leishmaniose cutânea americana. Alimentação sanguínea. Precipitina.

\section{ABSTRACT}

The aim of this study was to determine the sources of blood meals for females of Lutzomyia whitmani, a phlebotomine species incriminated as the main vector for American cutaneous leishmaniasis in Maranhão. For this, 70 Lutzomyia whitmani females were collected in the municipality of Axixá, an area with one of the greatest numbers of cases of American cutaneous leishmaniasis in humans in Maranhão. They were analyzed using the precipitin technique. Ninety percent of the specimens showed a reaction to some type of antiserum positive immune reaction, among which $73 \%$ presented single reactions, with predominance for chicken blood (22\%), rodent blood (14.3\%) and human blood (12.7\%). Among the double reactions, the predominant combinations were chicken/human (6.3\%), chicken/opossum (4.8\%), ox/human (3.2\%) and opossum/human (3.2\%). Thus, we conclude that humans and domestic and synanthropic animals are blood meal sources for Lutzomyia whitmani and may play an important role in the transmission cycle for American cutaneous leishmaniasis, thus explaining the cases of this disease in Axixá.

Key-words: Lutzomyia whitmani. American cutaneous leishmaniasis. Blood feeding. Precipitin.

Lutzomyia whitmani (Diptera, Psychodidae) é uma espécie de flebotomíneo incriminada em muitas regiões como principal vetor de espécies de Leishmania causadoras da leishmaniose cutânea americana (LCA $)^{6}$. Dentre as espécies que parasitam o

1. Programa de Pós Graduação em Biodiversidade e Conservação, Universidade Federal do Maranhão, São Luis, MA. 2. Laboratório de Entomologia e Vetores, Universidade Federal do Maranhão, São Luis, MA. 3. Departamento de Entomologia, Instituto Oswaldo Cruz, Fundação Oswaldo Cruz, Rio de Janeiro, RJ. 4. Departamento de Biologia, Universidade Federal do Maranhão, São Luis, MA.

Endereço para correspondência: Prof. José Manuel Macário Rebêlo. Laboratório de Entomologia e Vetores/Dept ${ }^{\underline{o}}$ de Patologia/UFMA. Praça Madre Deus 2, 65025-560 São Luis, MA

Tel: $55982108-8542$

e-mail: macariorebelo@uol.com.br

Recebido para publicação em 10/03/2009

Aceito em 08/10/2009 homem, podemos citar Leishmania braziliensis (Kinetoplastida, Trypanosomatidae), no Nordeste e Sudeste e Leishmania shawi (Kinetoplastida, Trypanosomatidae) na Região Norte ${ }^{7}$

Nos últimos anos, Lutzomyia whitmani vem sendo utilizada freqüentemente em estudos experimentais relacionados à taxa de infecção por Leishmania, o que proporciona um melhor conhecimento desta como vetor ${ }^{10}$. Apesar de Lutzomyia whitmani ser frequentemente encontrada em abrigos de animais domésticos, pouco se conhece a respeito de seu comportamento alimentar.

A atração que diferentes animais silvestres e domésticos exercem sobre os flebotomíneos como fonte alimentar constitui-se importante para o conhecimento das relações hospedeiro-vetor nos diversos ambientes, sobretudo em áreas com transmissão de leishmanioses. 
As ações humanas sobre o meio ambiente atuam na seleção das espécies de flebotomíneos e mamíferos reservatórios de Leishmania, permitindo àqueles com maior valência ecológica se adaptarem ao ambiente antrópico. Essas ações parecem favorecer a presença desses insetos e mamíferos no domicílio e peridomicílio, explicando, em parte, a persistência das leishmanioses nesse tipo de ambiente. Além disso, as habitações humanas de má qualidade e em locais inadequados, a construção desordenada de abrigos de animais domésticos no ambiente peridomiciliar e a carência de condições mínimas de saneamento básico são condições comuns em áreas rurais e periféricas de centros urbanos ${ }^{8}$. Nessas áreas, os mamíferos reservatórios de Leishmania têm sobrevivido e os flebotomíneos têm sido capturados em grande número ${ }^{11}$ favorecendo a infecção humana e de animais domésticos.

Os estudos das fontes sanguíneas de flebotomíneos têm sido muito úteis no esclarecimento da epidemiologia das leishmanioses, podendo vir a auxiliar as atividades de controle e vigilância dessas doenças ${ }^{4}$.

Assim, o estudo do conteúdo estomacal de insetos hematófagos é de grande significado ecológico e epidemiológico, pois além de permitir descobrir a identidade dos hospedeiros que servem de alimento aos flebotomíneos, por ocasião do repasto sanguíneo, pode fornecer subsídios para a indicação de reservatórios de Leishmania.

Neste trabalho, estuda-se a fonte alimentar sanguínea de uma população de Lutzomyia whitmani com o intuito de ampliar os conhecimentos sobre possíveis reservatórios de Leishmania em uma das áreas com maior número de casos de LCA em humanos no Maranhão, onde estudos prévios sobre taxa de infecção natural e levantamento de casos de LCA demonstraram a presença do parasita nos flebotomíneos e de grande quantidade de pessoas contaminadas revelando que há infecção circulando na região5.

\section{MATERIAL E MÉTODOS}

Área de estudo. As coletas foram realizadas no Município de Axixá ( $2^{\circ} 50^{\prime} 08^{\prime \prime}$ S e $44^{\circ} 03^{\prime} 14^{\prime \prime}$ W), localizado na região norte do Estado do Maranhão e sua cobertura vegetal inclui o manguezal e a restinga.

O clima é tropical mesotérmico e úmido e os índices pluviométricos podem atingir $2.000 \mathrm{~mm} / \mathrm{ano}$. As temperaturas são elevadas o ano todo (média de $27^{\circ} \mathrm{C}$ ), com variação anual pequena. Os locais de coleta foram selecionados no peridomicílio de bairros com maior número de casos de LCA e características essencialmente rurais, o que permitiu o encontro do vetor em frequências favoráveis à realização do trabalho.

Os espécimes foram capturados com uso de quatro armadilhas luminosas em miniatura tipo CDC (Center Control Disease), alimentadas por 4 pilhas alcalinas grandes de 1,5 volts, que foram instaladas no peridomicílio de 10 casas rurais. Estas funcionaram das 18:00h às 06:00h, ininterruptamente, uma vez por mês durante 10 meses. Os insetos retidos foram triados com auxílio de esteromicroscópio para separação de fêmeas ingurgitadas de
Lutzomyia whitmani que foram confirmadas quanto a espécie através da dessecação dos 2 últimos segmentos abdominais e identificação da espermateca seguindo chave dicotômica ${ }^{14}$.

Para determinação das fontes alimentares sanguíneas, os insetos foram analisados pelo teste da reação da precipitina na FIOCRUZ, Rio de Janeiro, onde o tubo digestório dos espécimes foi retirado e o conteúdo estomacal foi triturado em salina a $0,8 \%$. Este macerado foi deixado por 12 horas à temperatura entre 4 e $8^{\circ} \mathrm{C}$ e $\log 0$ após, centrifugado por 5 minutos a 1.500rpm, e o sobrenadante foi exposto a antisoros de humano, galinha, boi, cão, cavalo, gato, porco, roedor, gambá, tatu, lagarto e sapo.

\section{RESULTADOS}

No total, foram examinadas 70 fêmeas de Lutzomyia whitmani e $90 \%$ apresentaram reação aos diversos antissoros utilizados neste experimento.

Das fêmeas que apresentaram reação a algum tipo de antissoro, cerca de $73 \%$ foi do tipo simples, ou seja, reagiram a apenas um único antissoro e $27 \%$ apresentaram reações duplas, reagindo a dois antissoros de combinações diversas. No primeiro caso, as fêmeas reagiram a 6 diferentes tipos de antissoro e no segundo a 10 combinações diferentes. Nas reações simples, antisoros de galinha, roedor e humano predominaram com $22,2 \%, 14,3 \% \mathrm{e}$ 12,7\%, respectivamente (Tabela 1). Já nas reações duplas, as combinações galinha/humano (6,3\%) e galinha/gambá (4,8\%) se sobressaíram diante das demais (Tabela 1).

Levando em consideração os dois tipos de reação, as galinhas foram o tipo de fonte alimentar mais procurada pelos insetos com $28,7 \%$ do total de fêmeas positivas para algum tipo de antissoro (Figura 1).

TABELA 1

Relação entre número de fêmeas de Lutzomyia whitmani e reação aos diferentes tipos de antissoros.

\begin{tabular}{lrr}
\hline & \multicolumn{2}{c}{ Fêmeas } \\
\cline { 2 - 3 } Antissoro & $\mathrm{n}^{\mathbf{0}}$ & $\%$ \\
\hline Galinha & 14 & 22,2 \\
Humano & 8 & 12,7 \\
Porco & 4 & 6,3 \\
Gambá & 7 & 11,1 \\
Roedor & 9 & 14,3 \\
Boi & 4 & 6,3 \\
Gambá/porco & 1 & 1,6 \\
Galinha/gambá & 3 & 4,8 \\
Galinha/boi & 1 & 1,6 \\
Galinha/humano & 4 & 6,3 \\
Galinha/roedor & 1 & 1,6 \\
Boi/gambá & 1 & 1,6 \\
Boi/humano & 2 & 3,2 \\
Gambá/humano & 2 & 3,2 \\
Gambá/roedor & 1,6 \\
Boi/roedor & 1 & 1,6 \\
Total & 1 & 1,6 \\
\hline
\end{tabular}




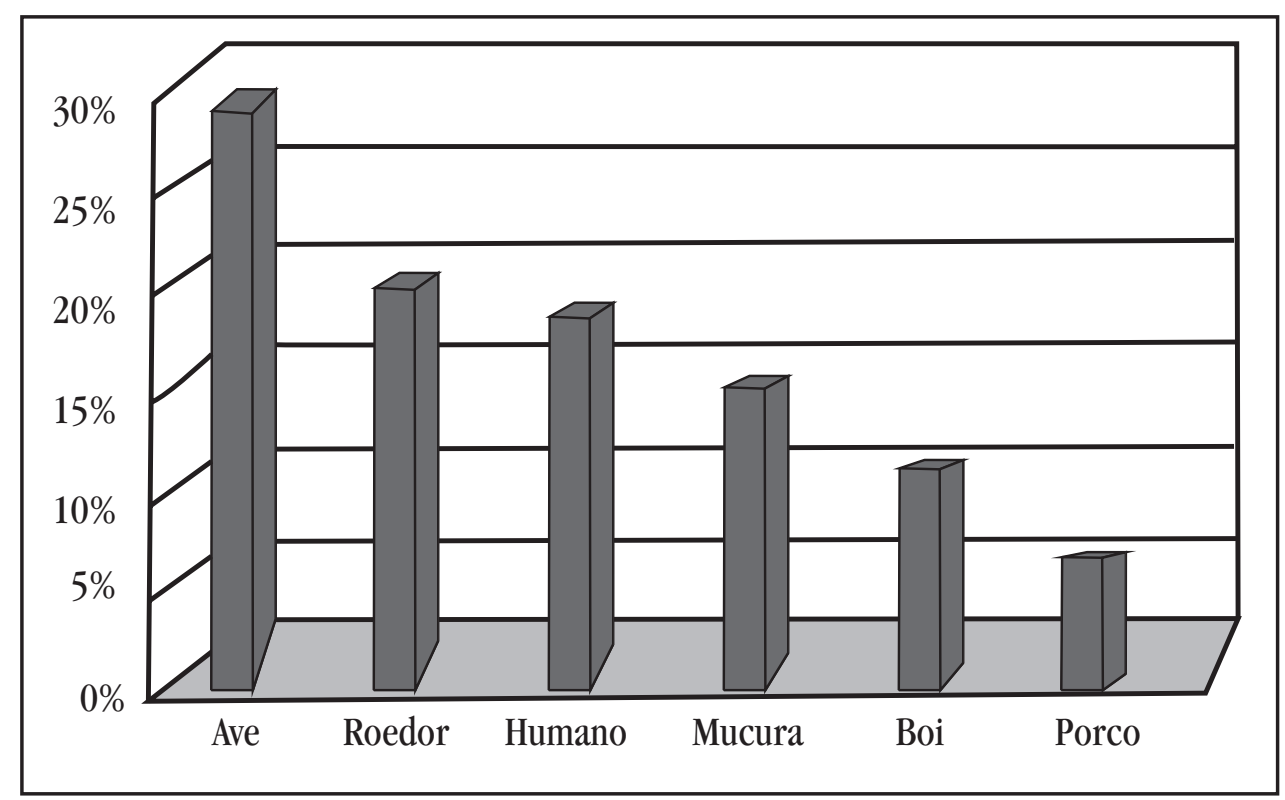

FIGURA 1

Preferência alimentar de fêmeas de Lutzomyia whitmani do município de Axixá.

\section{DISCUSSÃo}

Investigar o conteúdo estomacal de insetos hematófagos constitui-se em um importante estudo principalmente quando se trata de espécies incriminadas na transmissão de microorganismos patogênicos com é o caso de Lutzomyia whitmani.

Utilizando-se da reação da precipitina como metodologia para detectar a fonte alimentar sanguínea, neste trabalho observou-se que Lutzomyia whitmani possui um comportamento bastante eclético, uma vez que sugou o sangue de galinhas e mamíferos domésticos e sinantrópicos, incluindo ainda na sua dieta, o sangue humano. 0 caráter oportunista desta espécie já foi verificado por outros autores $^{2}{ }^{12}$ e constitui um aspecto ecológico de grande relevância na epidemiologia da LCA.

A análise do conteúdo estomacal de fêmeas de flebotomíneos, por qualquer um dos métodos utilizados (precipitina, ELISA ou dot-ELISA), tem mostrado que os flebotomíneos, de modo geral, ajustam o seu padrão alimentar de acordo com a disponibilidade de hospedeiros, mostrando o oportunismo desses dípteros que sugam uma ampla variedade de vertebrados ${ }^{13}$.

A presença de animais domésticos e a possibilidade deles virem a participar como hospedeiros sanguíneos dos flebótomos podem favorecer a aproximação e a manutenção destes insetos no peridomicílio ${ }^{4}$.

Neste estudo realizado em Axixá, verificou-se que nas casas onde não havia abrigo de animais domésticos, as capturas não eram bem sucedidas, caracterizando que a presença de Lutzomyia whitmani está diretamente relacionada à presença destes animais.

Notavelmente, Lutzomyia whitmani tendeu a sugar mais sangue de galinha neste estudo, uma vez que este grupo predominou em relação aos demais nas reações duplas e simples. Isto provavelmente pode ser explicado pela grande quantidade de casas com galinheiros no município e Axixá. Esse resultado mostra a grande influência atrativa que estas galinhas têm em relação à fonte de alimentação de Lutzomyia whitmani.

Por outro lado, no município de Buriticupu, na região amazônica do estado, a oeste, Lutzomyia whitmani apresentou maior associação com sangue de roedores $(44,4 \%)$ e de equinos $(22,2 \%)$ e menos sangue humano $(11,1 \%)$, apesar das galinhas (galinhas) estarem presentes em $87 \%$ das residências 9 .

Um forte indício da atratividade que as galinhas exercem sobre os flebotomíneos foi observado na Vila Bom Viver, município da Raposa localizado na Ilha de São Luis, MA, quando a presença de Lutzomyia longipalpis (Diptera, Psychodidae), incriminado como vetor da Leishmania chagasi (Kinetoplastida, Trypanosomatidae) causador da leishmaniose visceral estava diretamente relacionado com a presença de galinhas no peridomicílio das casas onde foram realizadas as coletas ${ }^{4}$.

Apesar da literatura não apresentar nenhum relato de que galinhas sejam reservatórios de Leishmania ${ }^{4}$, sugere que esta galinha tenha um papel muito importante na peridomiciliação do Lutzomyia longipalpis e Lutzomyia whitmani, pois funcionam como chamariz para os vetores, mantendo-os no ambiente humano. Este aspecto foi verificado em estudos realizados nos Municípios da Raposa ${ }^{1}$ e de São José de Ribamar ${ }^{3}$, na Ilha de São Luís, quando uma grande quantidade de Lutzomyia longipalpis foram capturados no ambiente peridoméstico, utilizando-se a galinha como isca.

O segundo grupo de animais mais sugados pelas fêmeas de Lutzomyia whitmani foi o dos roedores, considerados animais sinantrópicos. A presença destes vertebrados no peridomicílio como visitantes frequentes foi relatada pelos moradores da região onde foram realizadas as coletas. Este fato mostra a grande necessidade de estudar estes animais como possíveis reservatórios da Leishmania.

Um dado importante a ser observado é a ausência de reação para antissoro de cães apesar da presença destes no peridomicílio das casas onde foram realizadas as coletas mostrando a 
preferência das fêmeas de Lutzomyia whitmani a vertebrados domésticos como as galinhas (galinhas) e a animais sinantrópicos ao peridomicílio como roedores e gambás.

Outro fato que merece grande destaque é a presença do homem como terceiro grupo mais utilizado pelas fêmeas para o repasto sanguíneo, sendo superado somente pelas galinhas e roedores, demonstrando relativa proximidade de Lutzomyia whitmani com o ambiente antropizado. Além disso, o homem esteve presente em reações duplas com galinhas, boi e gambá onde, este último, pode ser o potencial reservatório da Leishmania, uma vez que existem casos positivos da doença na região das coletas. 0 mesmo pode ser observado em um trabalho desenvolvido na Costa Rica, onde foi capturado um número significativo de flebótomos em iscas humanas ${ }^{15}$.

A presença de exemplares de Lutzomyia whitmani alimentados, ao mesmo tempo, com sangue humano e gambá no peridomicílio nos leva a crer na hipótese de que a transmissão da infecção esteja ocorrendo nos ambientes peri e intradomicílio, ou seja, todos os elos da cadeia de transmissão da Leishmania estão presentes nestes ambientes no Município de Axixá, o que pode explicar o número de casos de leishmaniose cutânea americana que vêm sendo notificados nos últimos anos.

\section{AGRADECIMENTOS}

Os autores agradecem à Coordenação de Aperfeiçoamento de Pessoal de Nível Superior (CAPES) e a Fundação de Amparo a Pesquisa e ao Desenvolvimento Científico e Tecnológico do Maranhão (FAPEMA) pelas bolsas de Mestrado concedidas.

\section{REFERÊNCIAS}

1. Araújo JC, Rebêlo JMM, Carvalho ML, Barros VLL. Composição dos flebotomíneos (Diptera, Psychodidae) do Município da Raposa-MA, Brasil. Área endêmica de leishmanioses. Entomologia y Vectores 7: 33-47, 2000.

2. Camargo-Neves VLF, Gomes AC, Antunes JL. Correlação da presença de espécies de flebotomíneos (Diptera: Psychodidae) com registros de casos da Leishmaniose Cutânea Americana americana no estado de São Paulo, Brasil. Revista da Sociedade Brasileira de Medicina Tropical 35: 299-306, 2002.
3. Carvalho ML, Rebêlo JMM, Araújo JC, Barros VLL. Aspectos ecológicos dos flebotomíneos (Díptera, Psychodidae) do Município de São José de Ribamar, MA, Brasil. Área endêmica de leishmanioses. Entomologia y Vectores 7: 19-32, 2000 .

4. Dias FOP, Lorosa ES, Rebelo JMM. Fonte alimentar sangüínea e a peridomiciliação de Lutzomyia longipalpis (Lutz \& Neiva, 1912) (Psychodidae, Phlebotominae). Caderno de Saúde Pública 19: 1373-1380, 2003.

5. Fonteles RS. Ecologia de flebotomíneos (Díptera, Psychodidae) e infecção natural de Lutzomyia whitmani por Leishmania no município de Axixá - Maranhão, Brasil. [Monografia]. Curso de Ciências Biológicas da Universidade Federal do Maranhão, 2007.

6. Galati EAB. Flebotomíneos do Brasil. In: Rangel EF, Lainson R (eds) Morfologia e taxonomia. Fundação Oswaldo Cruz, Rio de Janeiro, p. 23-51, 2003

7. Lainson R, Shaw JJ, Silveira FT, Souza AAA, Braga RR, Ishikawa EAY. The dermal leishmaniases of Brazil, with special reference to the eco-epidemiology of the disease in Amazonia. Memórias do Instituto Oswaldo Cruz 89: 435-443, 1994.

8. Lima AP, Minelli L, Comunello E, Teodoro U. Distribuição da Leishmaniose Cutânea Americana por imagens de sensoriamento remoto orbital, no estado do Paraná, sul do Brasil. Anais Brasileiros de Dermatologia 77: 681-692, 2002.

9. Oliveira-Pereira YN, Moraes JLP, Lorosa EL, Rêbelo JMM. Preferência alimentar sanguínea de flebotomíneos da Amazônia do Maranhão, Brasil. Caderno de Saúde Pública 24: 2183-2186, 2008.

10. Oliveira-Pereira YN, Rebelo JMM, Moraes JLP, Pereira SRF. Molecular diagnosis of the natural infection rate due to Leishmania sp in sandflies (Psychodidae, Lutzomyia) in the Amazon region of Maranhão, Brazil. Revista da Sociedade Brasileira de Medicina Tropical 39: 540-543, 2006.

11. Teodoro U. Características ecológicas de Flebotomíneos (Díptera Psychodidae) em habitats antrópicos, município de Jussara, Paraná, Brasil. Revista da Sociedade Brasileira de Medicina Tropical 29: 625-626, 1996.

12. Teodoro U, Alberton D, Kühl JB, Santos ES, Santos DR, Santos AR, Oliveira O, Silveira TGV, Lonardoni MVC. Ecologia de Lutzomyia (Nyssomyia) whitmani em área urbana do município de Maringá, Paraná. Revista de Saúde Pública 137: 651-656, 2003

13. Velo E, Paparisto A, Bongiorno G, Muccio T, Khoury C, Bino S, Gramiccia M, Gradoni L, Maroli M. Entomological and parasitological study on phlebotomine sandflies in central and northern Albania. Parasite 12: 45-49, 2005.

14. Young DG, Duncan MA. Guide to the identification and geographic distribution of Lutzomyia sand flies in Mexico, the West Indies; Central and South America (Diptera: Psychodidae). Memories of the American Entomology Institute 54: $1-881,1994$

15. Zeledon R, Murillo J, Gutierrez H. Observaciones sobre la ecologia de Lutzomya longipalpis (Lutz \& Neiva, 1912) y posibilidades de existencia de leishmaniasis visceral en Costa Rica. Memórias do Instituto Oswaldo Cruz 79: 455-459, 1984. 